\title{
Correction to: Multidisciplinary treatment for locally advanced breast cancer with internal mammary lymph node metastasis in an elderly patient
}

\author{
Haruko Takuwa $^{1}$ (1) $\cdot$ Wakako Tsuji $^{1}$ ' Yoshihiro Yamamoto ${ }^{2} \cdot$ Chikako Yamauchi $^{3} \cdot$ Fumiaki Yotsumoto $^{1}$
}

Published online: 3 September 2019

(c) The Japan Society of Clinical Oncology 2019

\section{Correction to: \\ International Cancer Conference Journal (2019) 8:1-6 https://doi.org/10.1007/s13691-018-0344-z}

In the original publication, in Table 1, the patient 3, 58 years of age, was scheduled to be administered eribulin after gemcitabine as adjuvant therapy. However, the patient was deceased before administration due to sepsis. Eribulin was not administered actually and it needs to be deleted. The corrected Table 1 is given in this Correction.

The original article can be found online at https://doi.org/10.1007/ s13691-018-0344-z.

Haruko Takuwa st24057@yahoo.co.jp

1 Department of Breast Surgery, Shiga General Hospital, 5-4-30, Moriyama-shi, Moriyama, Shiga 524-8524, Japan

2 Department of Pathology, Shiga General Hospital, Moriyama, Shiga, Japan

3 Department of Radiation Oncology, Shiga General Hospital, Moriyama, Shiga, Japan 
Table 1 Patient characteristics with internal mammary lymph node metastasis at diagnosis

\begin{tabular}{|c|c|c|c|c|c|c|c|c|}
\hline Age & Clinical stage & Location & Subtype & Neoadjuvant therapy & Operation & Radiation & Adjuvant therapy & Outcome \\
\hline 42 & T3N3bM0 & Upper-lateral & Luminal-HER2 & EC-wPTX + HCPT & $\mathrm{Bp}+\mathrm{SLNB}$ & $60 \mathrm{~Gy} / 30 \mathrm{fr}$ & $\mathrm{HCPT}+\mathrm{TAM}$ & SD \\
\hline 44 & $\begin{array}{l}\text { T4bN3bM1 (synchro- } \\
\text { nous bilateral) }\end{array}$ & Upper-mediolateral & Luminal-HER2 & $\mathrm{TC}$ & $\mathrm{Bt}$ & - & $\begin{array}{l}\text { TAM + zometa-DTX + } \\
\text { Per + HCPT + zometa }\end{array}$ & SD \\
\hline 58 & T4bN2bM0 (SCC) & Upper-medial & Luminal A-like & EC-wPTX-VNR & $\mathrm{Bp}+\mathrm{Ax}$ & $50 \mathrm{~Gy} / 25 \mathrm{fr}$ & DTX-TS-1 + ANA-GEM & $\begin{array}{l}\text { Death due } \\
\text { to sepsis }\end{array}$ \\
\hline 68 & $\mathrm{~T} 2 \mathrm{~N} 3 \mathrm{bM} 0$ & Lower-medial & Luminal B-like & wPTX & $\mathrm{Bp}+\mathrm{Ax}$ & $50 \mathrm{~Gy} / 25 \mathrm{fr}$ & LET & SD \\
\hline 82 & $\mathrm{~T} 2 \mathrm{~N} 3 \mathrm{bM} 0$ & Upper-medial & Luminal B-like & - & $\mathrm{Bp}+\mathrm{Ax}$ & $60 \mathrm{~Gy} / 30 \mathrm{fr}$ & UFT + LET & SD \\
\hline
\end{tabular}

Publisher's Note Springer Nature remains neutral with regard to jurisdictional claims in published maps and institutional affiliations. 